\title{
Mutual Coupling Reduction in Phased Array Antennas Applying High-Impedance Surface at X Band
}

José Bruno O. de Araújo ${ }^{1 *}$ (D), Vanessa P. R. Magri Souza² (D), Tadeu N. Ferreira ${ }^{2}$ (D), Leni J. de Matos $^{2}$ (I), Glaucio L. Siqueira ${ }^{1}$ (D) Jorge M. Souza ${ }^{1}$ (D), Maurício W. B. da Silva ${ }^{2}$ ${ }^{1}$ Department of Electrical Engineering, Center for Telecommunication Studies (CETUC), Pontifical Catholic University of Rio de Janeiro, Rio de Janeiro, Brazil, *dearaujo.jbruno@gmail.com, glaucio.siqueira@pucrio.br,mitrione@gmail.com

${ }^{2}$ Department of Telecommunication Engineering, Fluminense Federal University, Niterói, Brazil, vanessamagri@id.uff.br,tadeu_ferreira@id.uff.br,lenijm@id.uff.br, mauricio.weber@gmail.com

\begin{abstract}
This paper presents a microstrip phased array using High Impedance Surface - Electromagnetic Bandgap (HIS-EBG) in order to minimize the effects of mutual coupling among the antenna elements. Each element of the array is fed by a coaxial cable allowing the beam steering by controlling the signal of the input port of each element. This phased array operates at $X$ Band as well as the bandgap of the HIS-EBG structure. Its main applications are satellite communication and broadcasting systems. Results show reductions in the mutual coupling up to $12 \mathrm{~dB}$, and improvements in the return loss and gain.
\end{abstract}

Index Terms - electromagnetic bandgap, metamaterial, microstrip, phased array.

\section{INTRODUCTION}

The use of phased array antennas has been increasing in the last few years due to the fact that they present several advantages over the use of single antennas, such as the flexibility to steer the transmitted signal. Its applicability goes from radar for weather observation to the detection of targets [1]. Nevertheless, one of the major problems of this technology is related to the mutual coupling among antenna elements. This effect brings serious drawbacks to the system as a whole in terms of gain, return loss, and radiation efficiency, to name a few.

Meanwhile, Metamaterials (MTMs) have been used to overcome problems such as the ones cited above, hence improving antenna performance evaluation [2]-[4]. MTMs are periodic sub-wavelength structures that present unusual electromagnetic properties, which are not usually found in nature, such as materials with $\varepsilon$-negative (ENG), $\mu$-negative (MNG), and both $\varepsilon$ and $\mu$ negative (DNG). Hence, these features open the whole range of possibilities for their application in different fields of study [5].

Since the mutual coupling could affect the performance of the phased array in several aspects, different methods employing MTMs have been proposed to mitigate the mutual coupling and improve the return loss and gain, such as electromagnetic bandgap (EBG) [6], split-ring resonators (SRR) [7], and frequency-selective surfaces (FSS) [8]. In this paper, in order to minimize the mutual coupling, the concept of EBG is applied and analyzed. Furthermore, the EBG effect can be achieved employing 
different types of structures [9]. However, the use of a mushroom-like high impedance surface (HISEBG) to achieve the electromagnetic bandgap is one of the most efficient due to its compactness [10], which is essential to modern communication systems. Moreover, the HIS-EBG is classified as a type of metamaterial and one of its main roles is related to the creation of a bandgap that suppresses the propagation of surface waves at a certain frequency range [5].

The proposed microstrip array operates at $\mathrm{X}$ band $(8-12 \mathrm{GHz})$, which is used for military and civilian interests. Also, devices operating at $\mathrm{X}$ band are employed in satellite communications, broadcast systems, radar, and so on. Due to the importance of $\mathrm{X}$ band, phased arrays operating at this frequency band can be useful in several applications [11].

In this paper, a $3 \times 3$ phased array with HIS-EBG is proposed in order to avoid some negative effects of the mutual coupling at X Band. Furthermore, the proposed work is organized as follows. Section II discusses theoretical, design and simulation issues regarding HIS-EBG. The employment of HIS-EBG in phased array and discussion about the simulations are exposed in Section III. Finally, the conclusion is presented in Section IV.

\section{HIGH IMPEDANCE SURFACE-ELECTROMAGNETIC BANDGAP}

In this paper, a mushroom-like HIS-EBG is used, which is composed of metallic patches arranged periodically on a dielectric substrate with a metal sheet on the bottom side, which plays the role of a ground plane. Moreover, these patches and the ground plane are connected by metal-plated vias. This structure yields an electromagnetic bandgap that suppresses the surface waves to propagate on the structure. Also, if the periodicity of the unit cell is small enough when compared to the operating wavelength, the properties of this structure can be described using lumped inductors and capacitors, once they behave as a parallel resonant LC network, acting as an electric filter that blocks the current to flow along the sheet.

The proposed HIS-EBG structure is designed to operate at X-Band and its unit cell can be seen in Fig. 1, where $r$ is the radius of the via, $b$ is the length of the square patch and $g$ is the gap between the patch and the outer limit of the unit cell. The FR-4 substrate is used, which has a thickness of $1 \mathrm{~mm}$, a dielectric constant of 4.4 and a loss tangent of 0.02 , and the dimensions of the HIS-EBG at issue are $b$ $=2.600 \mathrm{~mm}, g=0.155 \mathrm{~mm}$ and $r=0.100 \mathrm{~mm}$. In addition, the proposed HIS-EBG can be considered to be sub-wavelength, once its periodicity is $2.775 \mathrm{~mm}$ or, in terms of the wavelength at the central frequency of the $\mathrm{X}$ band $(10 \mathrm{GHz}), 0.092 \lambda_{0}$.

The behavior of the HIS-EBG is analyzed using the dispersion relation between the frequency and the wavevector $k$, which is also known as the dispersion diagram. Moreover, this diagram can be plotted for the surface wave propagation in the HIS-EBG on HFSS using Eigenmode solver, which is used to find the natural resonances and dispersion properties of the structure at issue, and applying master/slave boundaries in the unit cell, as shown in Fig. 2. 


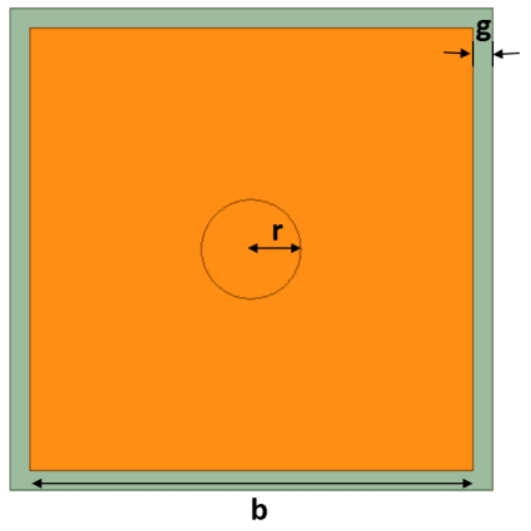

Fig. 1. Top view of the unit cell and its main parameters.

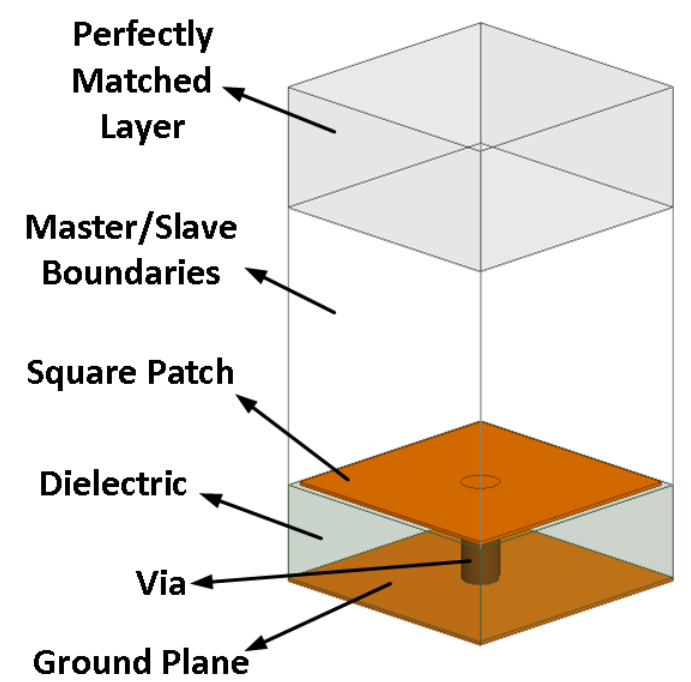

Fig. 2. Unit cell of the HIS on the HFSS.

The proposed HIS-EBG yields a dispersion diagram over the irreducible Brillouin zone in which the electromagnetic bandgap will be analyzed, as shown in Fig. 3. One can note in Fig. 3 (b) the Brillouin zone of a square patch, where $k_{\mathrm{x}}$ and $k_{\mathrm{y}}$ are the wavenumber in $\mathrm{x}$ and $\mathrm{y}$-direction, respectively. Moreover, due to the symmetry of the unit cell, the irreducible Brillouin zone can be represented by three points, which are $\mathrm{R}\left(k_{\mathrm{x}}=k_{\mathrm{y}}=0\right), \mathrm{M}\left(k_{\mathrm{x}}=\pi / \Lambda\right.$ and $\left.k_{\mathrm{y}}=0\right)$ and $\mathrm{X}\left(k_{\mathrm{x}}=0\right.$ and $k_{\mathrm{y}}=$ $\pi / \Lambda)$, where $\Lambda$ is the periodicity of the unit cell.

The dispersion diagram is plotted in Fig. 3 (a) considering the irreducible Brillouin zone of the unit cell at issue. In this graph, the blue and red solid lines represent the solution to their corresponding eigenvalue equations for the transverse electric (TE) and transverse magnetic (TM) modes, respectively, and, thus, the electromagnetic wave can propagate at frequencies with the solution. However, it is possible to observe that the proposed HIS-EBG structure provides an electromagnetic bandgap over the $\mathrm{X}$-band, i.e. there is no solution over this frequency range, which means that surface waves at frequencies within this gap are forbidden to propagate through the HIS-EBG [12]. This happens due to the interaction of the metal vias with the surface wave, which slows down the Brazilian Microwave and Optoelectronics Society-SBMO received 15 May 2019; for review 16 May 2019; accepted 9 Dec 2019 
propagation of the surface waves until their suppression [13]. The role of the patches is to attenuate the effects of spatial dispersion [14]. Furthermore, the gap is placed between the two fundamental orthogonal modes, which are the TE and TM modes.

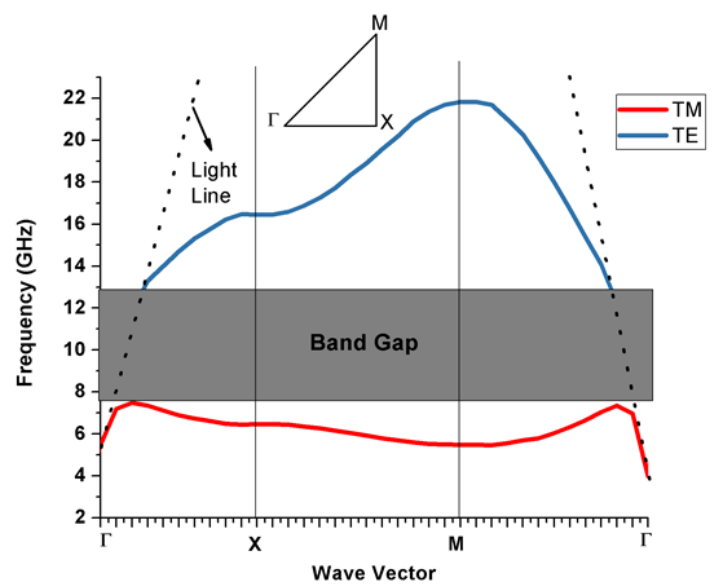

(a)

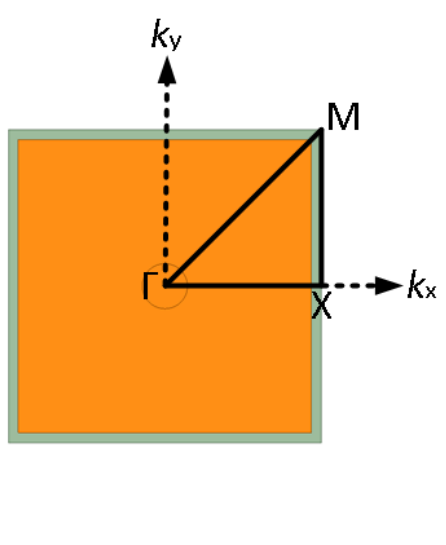

(b)

Fig. 3. Dispersion diagram (a) and irreducible Brillouin Zone (b) of the HIS-EBG unit cell.

This bandgap implies that surface waves at frequencies encompassed by the gap will not propagate through the HIS-EBG structure, and, then, it can be applied to arrays to reduce the mutual coupling among the antenna elements. Furthermore, the provided bandgap includes the operating frequency of the phased array, which is $10 \mathrm{GHz}$.

\section{PhASED ARRAY WITH HIS-EBG}

The proposed microstrip-based phased array is designed to operate at $10 \mathrm{GHz}$ and takes advantage of the EBG-HIS to decrease the mutual coupling among its antenna elements. Furthermore, each element of the array features a rectangular microstrip patch that uses coaxial probe-fed. This feeding technique provides an easy implementation, minimizes spurious radiation and reduces the size of the microstrip antenna when compared to other methods [15]. Besides that, each cell presents one row of HIS-EBG elements surrounding the patch, as it can be seen in Fig. 4 that shows the unit cell of the proposed phased array, where $W$ and $L$ are the width and length of the unit cell at issue, respectively, $w$ and $l$ are the width and length of the patch, and $h$ is the thickness of the substrate. The dimensions of the array, in mm, are $w=6.50, l=6.60, W=23.28, L=23.28$, and $h=1$. Also, the position of the feeding is indicated by $w^{\prime}$ and $l$ ' and their values, in mm, are 4.55 and 4.4 , respectively. 


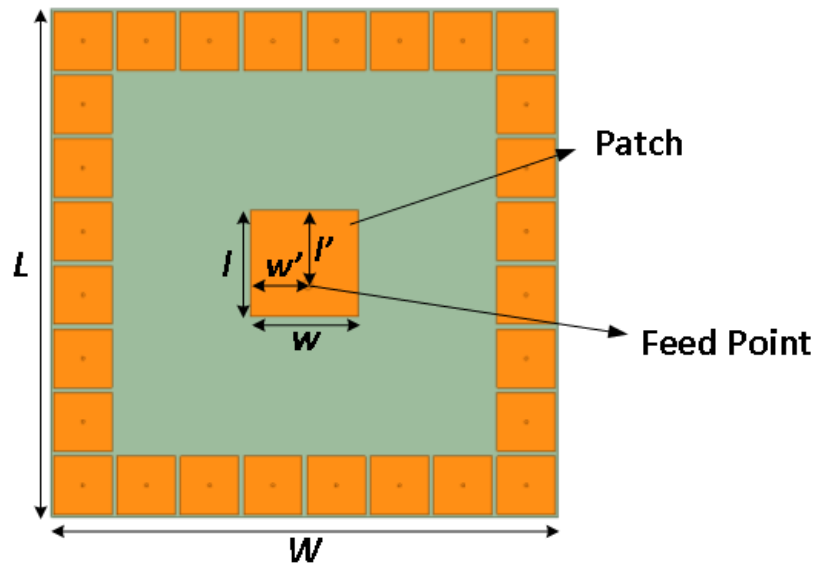

(a)

Substrate

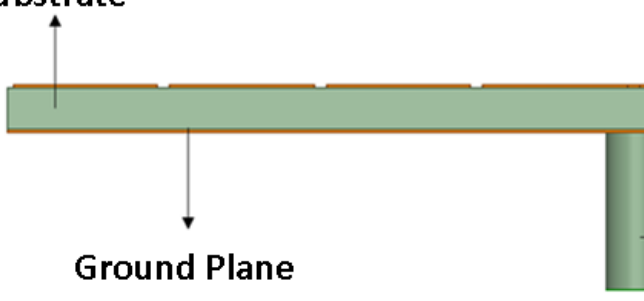

(b)

Fig. 4. Top (a) and side (b) views of phased array unit cell.

In order to provide a better understanding of the proposed structure, a $3 \times 3$ phased array is considered due to its symmetry, as can be seen in Fig. 5. The substrate used is FR-4 and the metal is the copper, and the dimensions of the array are $69.84 \mathrm{~mm} \times 69.84 \mathrm{~mm} \times 1 \mathrm{~mm}$. Also, it was used master/slave conditions in the unit cell of the phased array on HFSS. This simulation setup allows the employment of the Domain Decomposition Method (DDM), which allows the minimization of the simulation time and computational effort of the antenna array structure. In this method, the HFSS replicates the mesh of a single unit cell to create a finite antenna array. Thus, differently from most works in the literature that usually consider arrays with elements, in this work it was investigated the effects of the EBG in a phased array with 9 elements.

To highlight the influence of the presence of the HIS-EBG in the array, the reflection coefficients of the central element of the array with and without HIS-EBG structure are simulated on HFSS and plotted, as can be seen in Fig. 6. It is possible to observe that there is a slight upward shift, from 9.9 to $10 \mathrm{GHz}$, in the resonance frequency with the presence of HIS-EBG. Additionally, the value of the reflection coefficient varies with the HIS-EBG. To be more specific, for the phased array with HISEBG, the reflection coefficient at the central frequency is $-44.9 \mathrm{~dB}$, and for the phased array without HIS- EBG, $-27.1 \mathrm{~dB}$, which shows a significant improvement of this parameter with the employment of the electromagnetic bandgap structure. Furthermore, one can realize that the presence of the HISEBG does not negatively affect the bandwidth of the patch at issue, once the 10-dB fractional 
bandwidths with and without the HIS-EBG are $4.48 \%(9.80-10.25 \mathrm{GHz})$ and $4.63 \%(9.80-10.16$ $\mathrm{GHz}$ ), respectively.

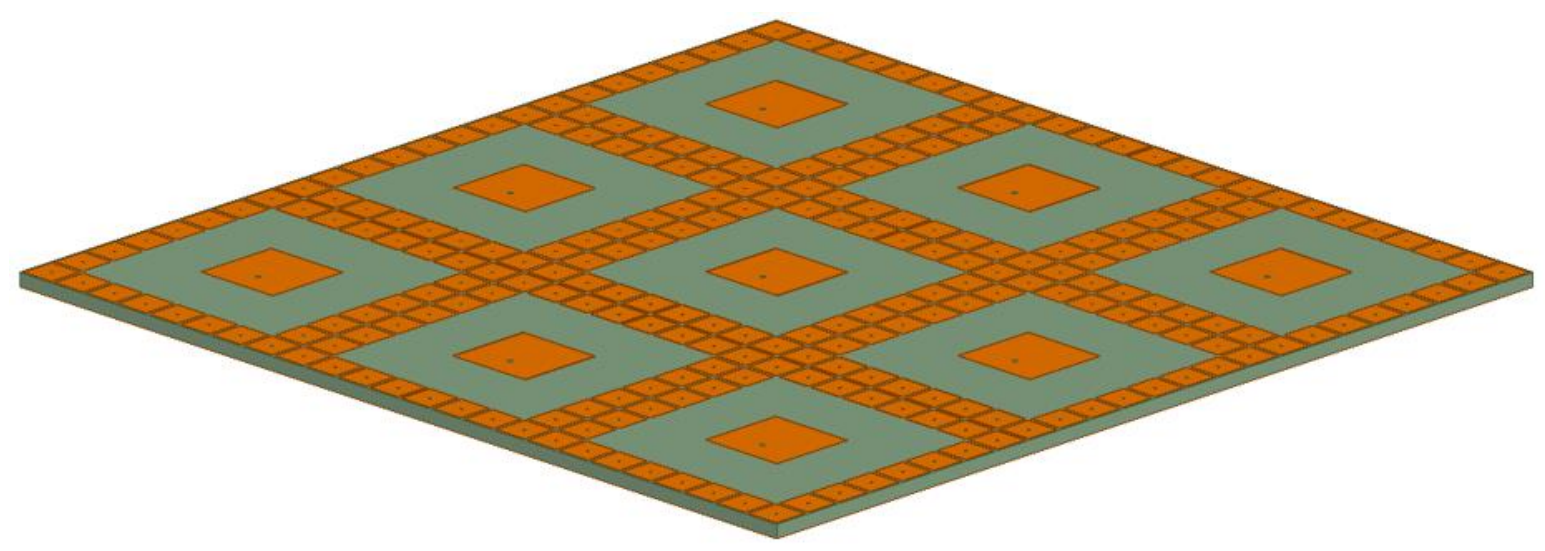

Fig. 5. The proposed $3 \times 3$ phased array with HIS-EBG.

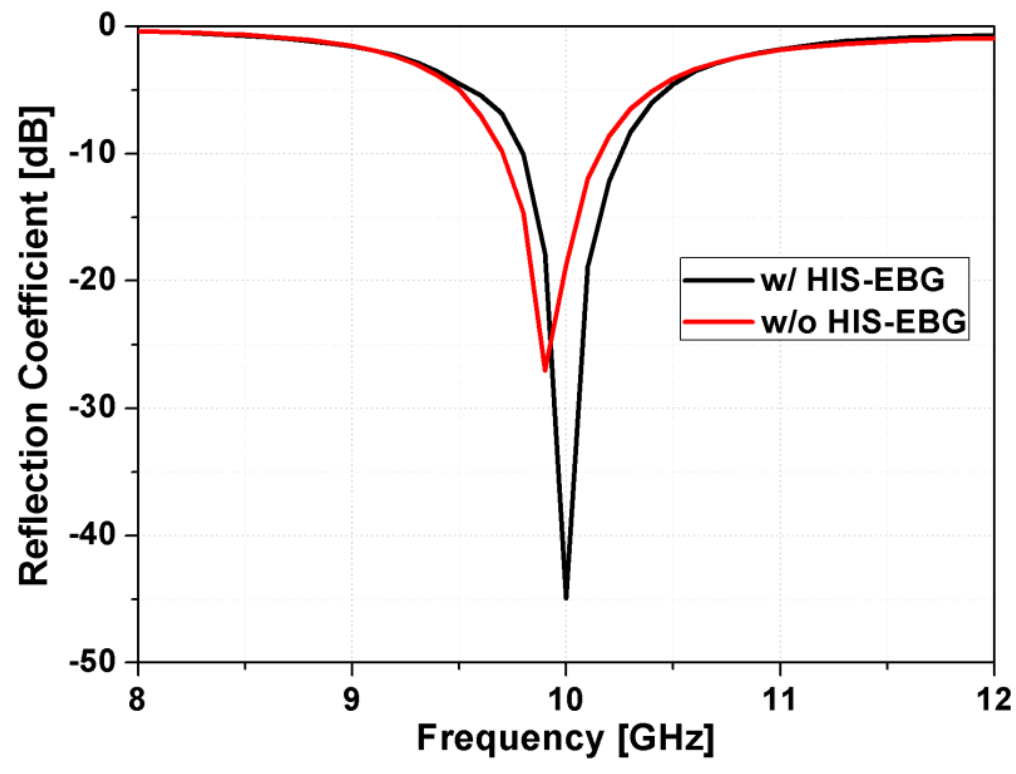

Fig. 6. Reflection coefficient of the phased array with and without HIS-EBG.

Even though the reflection coefficient provides an interesting perspective into the influence of the HIS-EBG on the central patch, it is important to present how the other elements behave. So, Fig. 7 shows the value of the reflection coefficient of each patch element at their respective resonant frequency considering the phased array with and without the bandgap structure. The patches in the top and bottom rows of the array present a slight increase in the reflection coefficient with the presence of the HIS-EBG. On the other hand, the patches at the central horizontal row present a significant improvement in the reflection coefficient. 


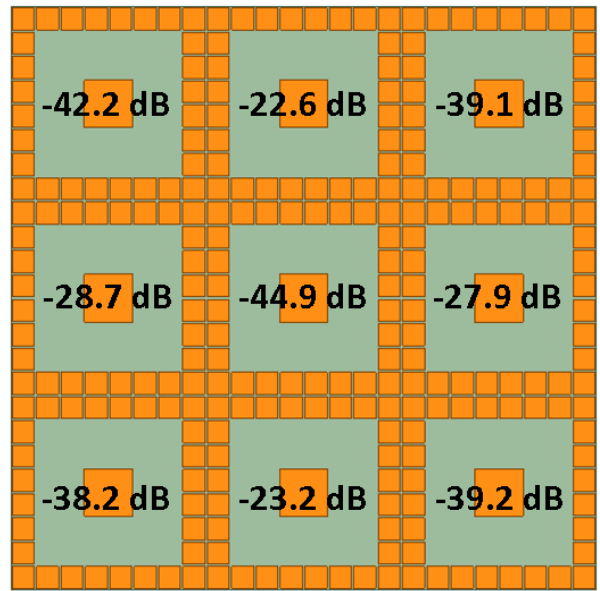

(a)

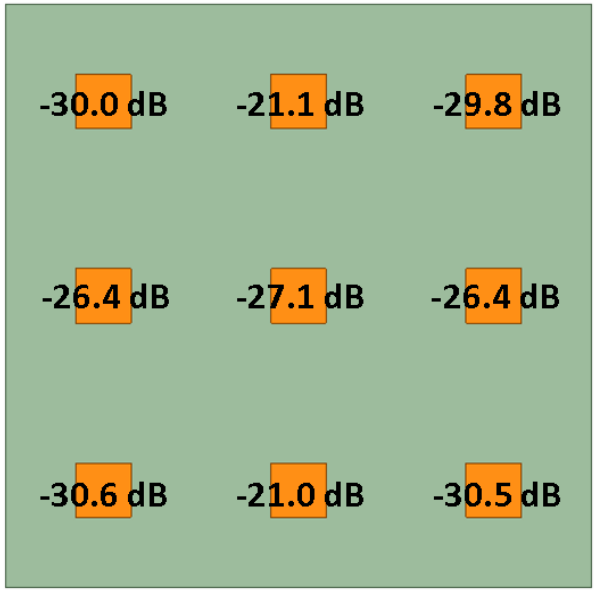

(b)

Fig. 7. Reflection coefficient of each patch element of the phased array with (a) and without (b) HIS-EBG.

In order to analyze the effects of HIS on the phased array, a comparison of the mutual coupling between the arrays with and without HIS-EBG is provided. Furthermore, the mutual coupling is reported in terms of the scattering parameters $\left(S_{\mathrm{XY}}\right)$ from the central element to the adjacent ones for the sake of the symmetry, which can be seen in Fig. 8, where only the central patch is being fed and $X$ denotes the central port and $Y$ represents the adjacent ones. The presence of HIS-EBG provides attenuation on the mutual coupling in all elements of the array and reductions up to $12.2 \mathrm{~dB}$, which means that the HIS-EBG structure is providing an enhancement of the isolation among the antennas. Moreover, it is possible to observe that the values of mutual coupling are not perfectly symmetric with respect to the central patch. This effect occurs due to the fact that the feeding point is not positioned at the center of the patch, which justifies the lack of symmetry.

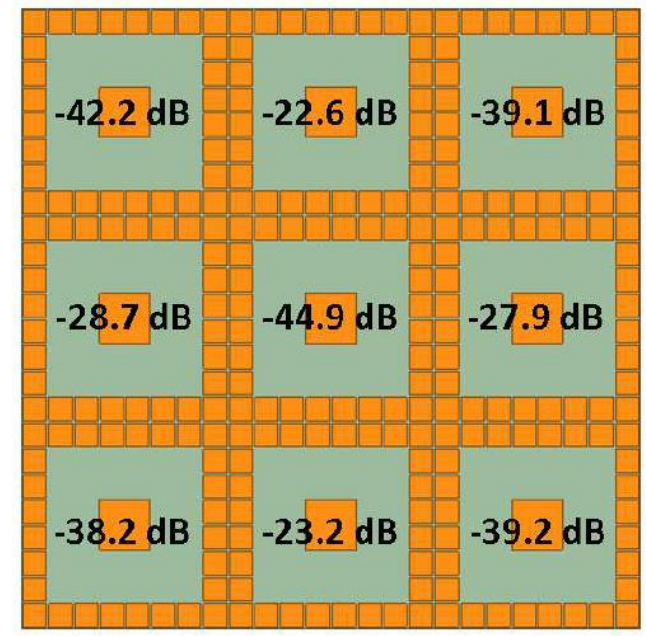

(a)

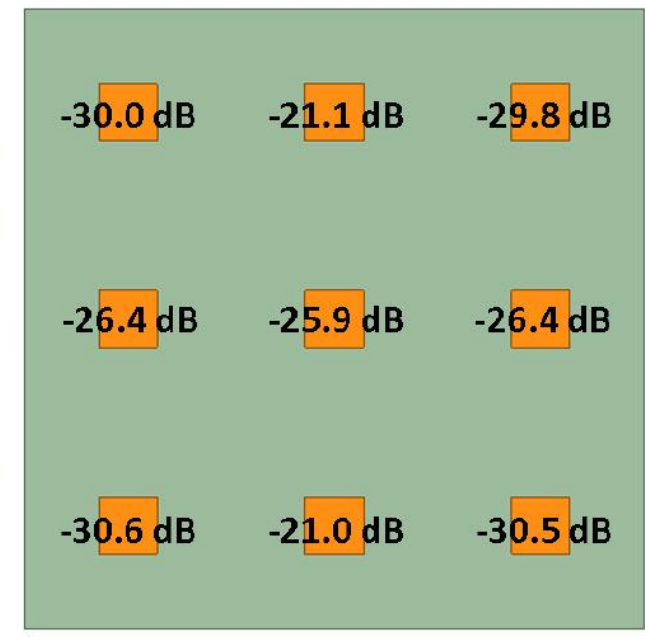

(b)

Fig. 8. Mutual coupling between the central element of the phased array to the others with (a) and without (b) HIS-EBG.

When it comes to the coupling among multiple antennas, it is essential to analyze the surface current distribution of the array. Fig. 9 shows the surface current distribution at $10 \mathrm{GHz}$ and $9.9 \mathrm{GHz}$ Brazilian Microwave and Optoelectronics Society-SBMO received 15 May 2019; for review 16 May 2019; accepted 9 Dec 2019 
for the phased array with and without HIS-EBG, respectively, in which only the central port is receiving an input signal. Furthermore, it is possible to realize that there is a strong current coupling from the central patch to the others on the phased array without HIS-EBG. However, with the employment of HIS-EBG, the induced current on the other patches decreases significantly. This happens because the HIS-EBG blocks the surface current to go from the central patch to the other ones, and, then, minimizing the mutual coupling.

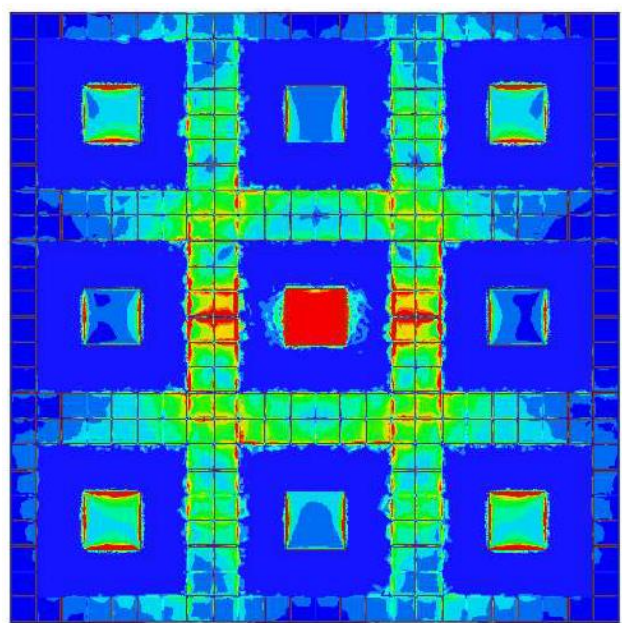

(a)
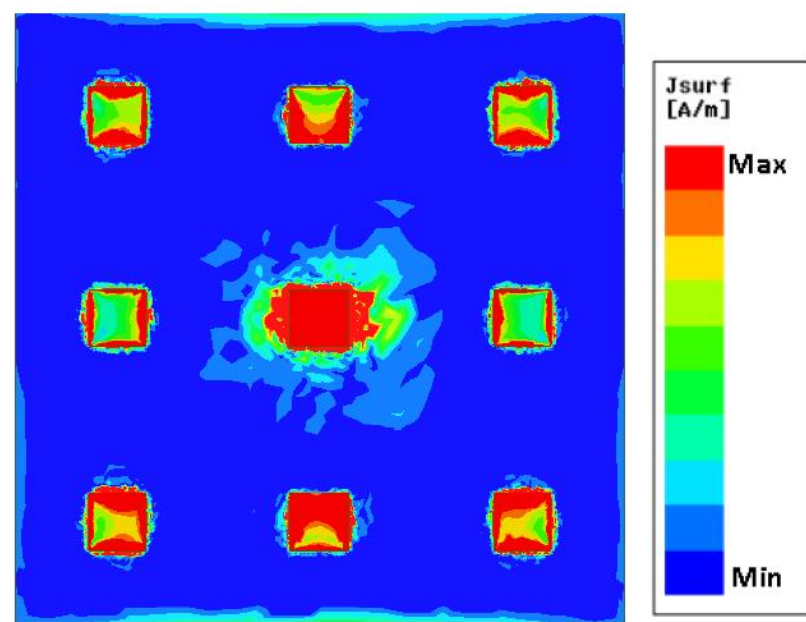

(b)

Fig. 9. Surface current distribution of the phased array with (a) and without (b) HIS-EBG.

Fig. 10 (a) and (b) show the gain patterns of the phased array with and without HIS-EBG, respectively. The radiation pattern was computed for the central patch considering all the other ports matched, i.e. only the central patch is being fed. However, there are changes in the half-power beamwidth (HPBW), which are $81.48^{\circ}$ and $133.51^{\circ}$ for the phased array with and without the bandgap, respectively. Also, the maximum gains are $5.41 \mathrm{dBi}$ and $4.03 \mathrm{dBi}$ for the array with and without the presence of the metamaterial. 


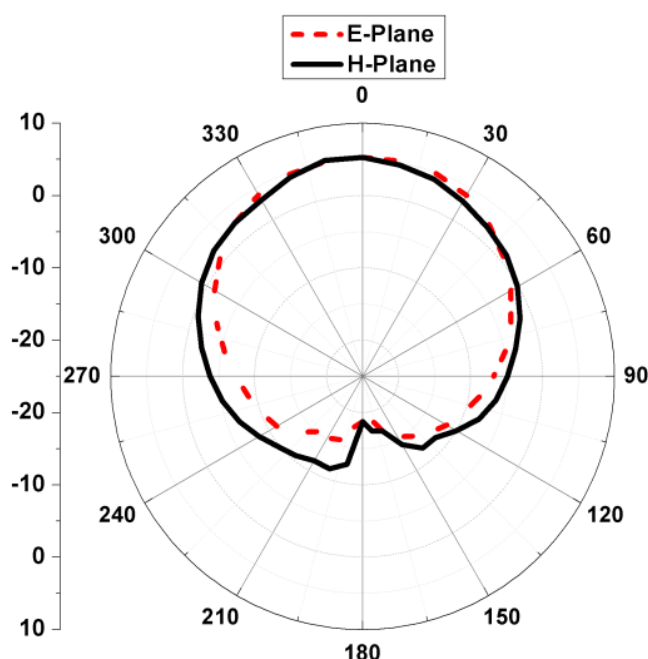

(a)

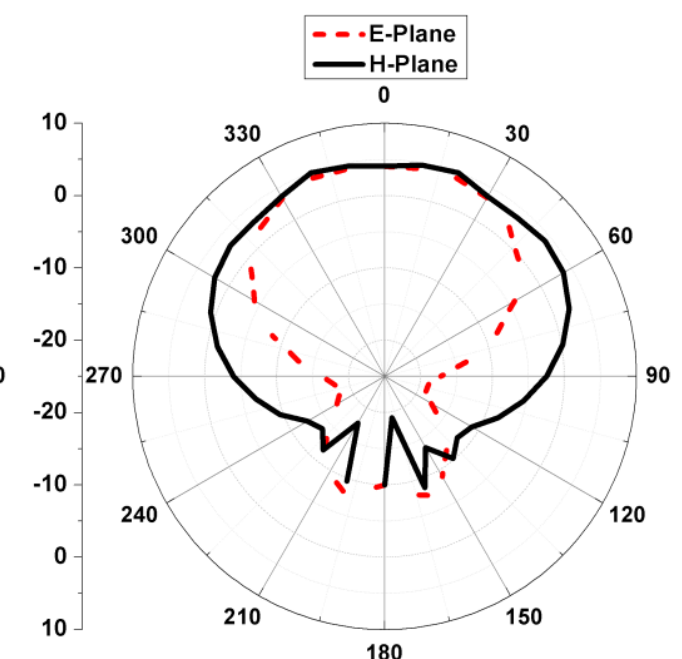

(b)

Fig. 10. Gain radiation patterns of the central patch with (a) and without (b) HIS-EBG.

TABLE 1 - PARAMETERS OF THE PROPOSED PHASED ARRAY WITH DIFFERENT INPUT CONFIGURATIONS.

\begin{tabular}{|c|c|c|c|c|}
\hline \multirow{2}{*}{ Configuration } & \multicolumn{2}{|c|}{ Half-Power Beam width } & \multicolumn{2}{c|}{ Main Lobe Direction } \\
\cline { 2 - 5 } & Plane $\mathbf{X Z}$ & Plane $\mathbf{Y Z}$ & $0^{\circ}$ & $\boldsymbol{\Phi}$ \\
\hline Fig. 11 (a) & $81.48^{\circ}$ & $82.89^{\circ}$ & $0^{\circ}$ & $0^{\circ}$ \\
\hline Fig. 11 (b) & $21.23^{\circ}$ & $22.55^{\circ}$ & $20^{\circ}$ & $90^{\circ}$ \\
\hline Fig. 11 (c) & $23.34^{\circ}$ & $21.86^{\circ}$ & $20^{\circ}$ & $270^{\circ}$ \\
\hline Fig. 11 (d) & $23.36^{\circ}$ & $21.20^{\circ}$ & & \\
\hline
\end{tabular}

One of the main features of the phased array is its capacity to steer the beam, change the beamwidth and shift the direction of the main lobe. This effect is achieved by changing the magnitude and phase of the input signal in each port of each element of the phased array. The tool HFSS' Finite Array Beam Angle Calculator is used to establish the phase shift necessary to steer the beam in a given direction. To illustrate that the beam steering features of the proposed structure are preserved even with the presence of the HIS-EBG, Fig. 11(a) shows the 3D radiation pattern of the HIS phased array with an input configuration that provides wide angular coverage, Fig. 11(b), the input signals in each port are combined in such way to create a more directive beam. Also, Fig. 11(c) denotes the phased array with the beam directed to $\theta=20^{\circ}$ and $\phi=90^{\circ}$. On the other hand, Fig. 11(d) shows the proposed structure configured so that the main lobe points to $\theta=20^{\circ}$ and $\phi=270^{\circ}$. The peak gains of the radiation patterns of Fig. 11(a), Fig. 11(b), Fig. 11(c), and Fig. 11(d) are, in dBi, 5.41, 14.93, 14.32, and 14.33, respectively. Also, Table I shows the HPBW for the radiation patterns showed in Fig. 11 for both $X Z$ and $Y Z$ planes and summarizes the main lobe direction. Therefore, the HIS-EBG structure does not compromise the main feature of a phased array, which is the beam steering. 


\section{CONCLUSION}

In order to minimize the effects of mutual coupling in a microstrip phased array, a mushroom-like HIS-EBG structure was design and simulated on HFSS to create an electromagnetic bandgap at the XBand, which has sub-wavelength dimensions. The proposed phased array uses a low-cost and lowprofile FR-4 substrate and operates at $10 \mathrm{GHz}$, which is encompassed by the stopband gap of HISEBG. The effectiveness of the employment of HIS-EBG was illustrated by comparing the surface current distribution, reflection coefficient, radiation patterns, and mutual coupling of the phased array with and without HIS-EBG. Reductions in mutual coupling up to $12 \mathrm{~dB}$ were found, which means an improvement of the insulation between the elements of the phased array employing HIS-EBG, as well as improvements up to $17.8 \mathrm{~dB}$ in the return loss. In addition, the presence of HIS-EBG improves reflection coefficient and mutual coupling, while maintains the radiation pattern characteristic of the phased array.

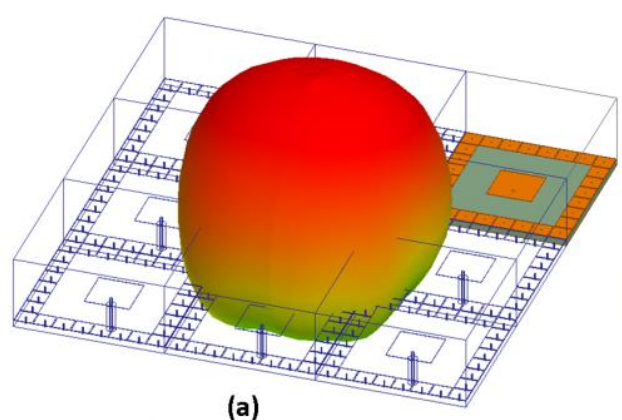

(a)

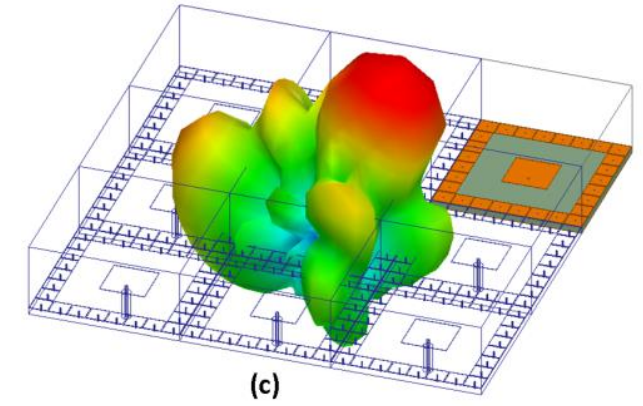

(c)

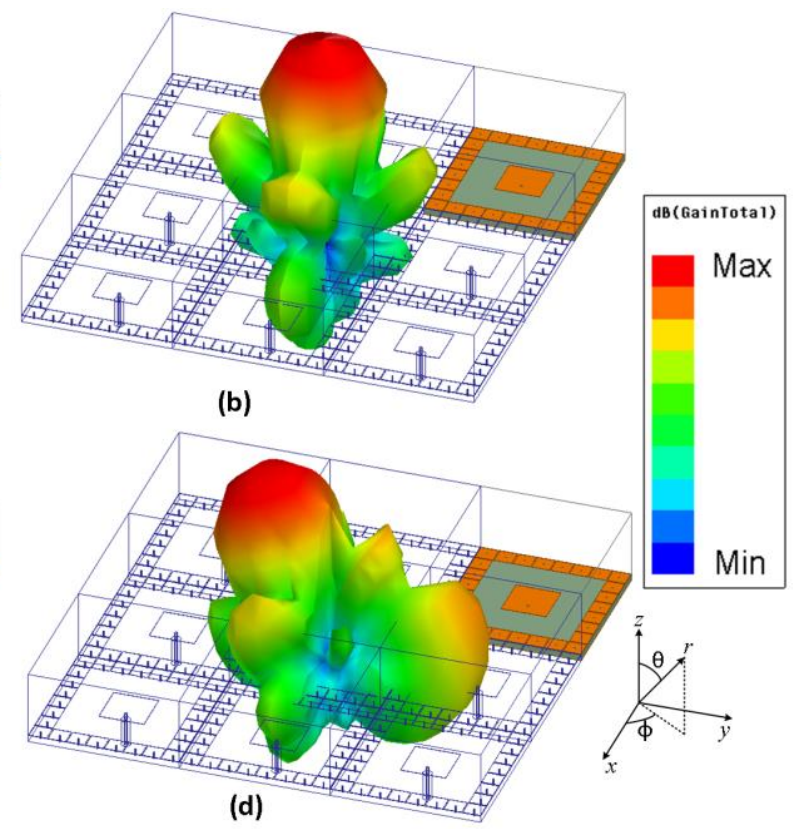

Fig. 11. 3D radiation patterns of the proposed phased array set for a larger (a) and directive (b) beam.

\section{REFERENCES}

[1] D. S. Zrnic, J. F. Kimpel, D. E. Forsyth, et al., "Agile-beam phased array radar for weather observations," Bulletin of the American Meteorological Society, vol. 88, no. 11, pp. 1753-1766, 2007.

[2] M. C. Tang, S. Xiao, B. Wang, J. Guan, T. Deng, "Improved performance of a microstrip phased array using broadband and ultra-low-loss metamaterial slabs," IEEE Antennas and Propagation Magazine, vol. 53, no. 6, pp. 31-41, 2011.

[3] A. Dadgarpour, B. Zarghooni, B. S. Virdee, T. A. Denidni, "Improvement of gain and elevation tilt angle using metamaterial loading for millimeter-wave applications," IEEE Antennas and Wireless Propagation Letters, vol. 15, pp. 418-420, 2016.

[4] S. Zhu, H. Liu, P. Wen, "A New Method for Achieving Miniaturization and Gain Enhancement of Vivaldi Antenna Array Based on Anisotropic Metasurface," IEEE Transactions on Antennas and Propagation, vol. 67, no. 3, pp. 19521956, 2019. 
Journal of Microwaves, Optoelectronics and Electromagnetic Applications, Vol. 19, No. 1, March 2020

DOI: http://dx.doi.org/10.1590/2179-10742020v19i11773

[5] N. Engheta, R. W. Ziolkowski, Metamaterials: physics and engineering explorations, John Wiley \& Sons, 2006.

[6] G. E. Dominguez, J. M. F. Gonzalez, P. Padilla, M. S. Castaner, "Mutual coupling reduction using EBG in steering antennas," IEEE Antennas and Wireless Propagation Letters, vol. 11, pp. 1265-1268, 2012.

[7] M. Suwailam, O. Siddiqui, O. Ramahi, "Mutual coupling reduction between microstrip patch antennas using slottedcomplementary split-ring resonators,” vol. 9, pp. 876-878, 2010.

[8] R. Karimian, A. Kesavan, M. Nedil, T. Denidni, "Low-mutual-coupling 60-GHz MIMO antenna system with frequency selective surface wall," IEEE Antennas and Wireless Propagation Letters, vol. 8, pp. 373-376, 2017.

[9] Y. Rahmat-Samii, H. Mosallaei, "Electromagnetic band-gap structures: classification, characterization, and applications," Proc. of 11th International Conference on Antennas and Propagation (ICAP 2001, pp. 560-564, 2001.

[10] D. Sievenpiper, L. Zhang, R. Broas, N. Alexopolous, E. Yablonovitch, "High-impedance electromagnetic surfaces with a forbidden frequency band," IEEE Transactions on Microwave Theory and Techniques, vol. 47, no. 11, pp. 2059-2074, 1999.

[11] E. Brookner, "Phased arrays around the world-progress and future trends," em IEEE International Symposium on Phased Array Systems and Technology, Boston, MA, 2003.

[12] F. Yang and Y. Rahmat-Samii, "Microstrip antennas integrated with electromagnetic band-gap (EBG) structures: A low mutual coupling design for array applications," IEEE Transactions on Antennas and Propagation, vol. 51, no. 10, pp. 2936-2946, 2003.

[13] D. Sievenpiper, "High-impedance electromagnetic surfaces," 1999.

[14] O. Luukkonen, M. G. Silveirinha, A. B. Yakovlev, C. R. Simovski, I. S. Nefedov and S. A. Tretyakov, "Effects of spatial dispersion on reflection from mushroom-type artificial impedance surfaces," IEEE Transactions on Microwave Theory and Techniques, vol. 57, no. 11, pp. 2692-2699, 2009.

[15] C. Balanis, Antenna theory: analysis and design, John Wiley \& Sons, 2016. 